\title{
Looking for Hypergiants in PeeringDB
}

\author{
Timm Böttger \\ Queen Mary University of London \\ timm.boettger@qmul.ac.uk
}

\author{
Felix Cuadrado \\ Queen Mary University of London \\ felix.cuadrado@qmul.ac.uk
}

\author{
Steve Uhlig \\ Queen Mary University of London \\ steve.uhlig@qmul.ac.uk
}

\begin{abstract}
Hypergiants, such as Google or Netflix, are important organisations in the Internet ecosystem, due to their sheer impact in terms of traffic volume exchanged. However, the research community still lacks a sufficiently crisp definition for them, beyond naming specific instances of them. In this paper we analyse PeeringDB data and identify features that differentiate hypergiants from the other organisations. To this end, we first characterise the organisations present in PeeringDB, allowing us to identify discriminating properties of the these organisations. We then use these properties to separate the data in two clusters, differentiating hypergiants from other organisations. We conclude this paper by investigating how hypergiants and other organisations exploit the IXP ecosystem to reach the global IPv4 space.
\end{abstract}

\section{CCS CONCEPTS}

- Networks $\rightarrow$ Public Internet; Very long-range networks; Network performance analysis;

\section{KEYWORDS}

Hypergiants, PeeringDB, Internet eXchange Points

\section{INTRODUCTION}

The Internet research community has commonly accepted that a significant fraction of today's Internet traffic relates to so-called hypergiants like YouTube or Netflix [5]. While their importance is known for some time now, the research community still falls short of a definition of hypergiants. Most evidence on their behaviour and existence is anecdotal, or self-reported but lacking sufficient detail $[3,11,12]$. The current way the community understands and defines hypergiants is mainly by naming examples, which we believe is unsatisfactory. This is surprising as hypergiants not only are a massive source of traffic, but they also are believed to be one of the driving forces behind the observed flattening of the Internet hierarchy. The reason for the observed flattening indeed is their approach to peering, reaching customers via direct peering links instead of using and paying transit providers. The amount of traffic they carry is so significant that it has shifted traffic away from the traditional hierarchy of the Internet, and thus asked the research community to revisit their mental model of the Internet [5].

To obtain a better understanding of the role hypergiants play in the Internet, we first analyse PeeringDB data to get a better understanding of the organisations taking part in public traffic exchange at IXPs. We then use the results of this analysis to identify features from the data available in PeeringDB to differentiate hypergiants from other organisations.

In this paper we make the following contributions:
(1) We characterise the organisations in PeeringDB, looking at several features: their geographical scope, provisioned port capacity and potential reach.

(2) We exploit a natural split in the data across those features to differentiate hypergiants from other organisations.

(3) We then explore how these hypergiants and other organisations reach the global IPv4 space through the IXP ecosystem.

Code and Data sharing. We make the PeeringDB data snapshot and code used for this paper available to the research community, in the hope that this stimulates and facilitates further research.

\section{PEERINGDB DATA SET OVERVIEW}

PeeringDB ${ }^{1}$ curates data to facilitate the exchange of information related to peering, by letting organisations and IXPs advertise themselves. In this paper we use the term organisation to refer to an entity participating in traffic exchange through the public Internet, with a record on PeeringDB. Google, Netflix and Yahoo are examples for organisations.

As of writing this paper, more than 600 IXPs and more than 10,000 organisations are present in PeeringDB. However, only 6,910 of them have at least one presence at a public IXP recorded. Refer to Table 1 for more details.

Data in PeeringDB is voluntarily reported by organisations and IXPs. Despite the self-reported nature of this data, making it potentially unreliable, its public nature and popularity for the Internet peering ecosystem guarantees that significant scrutiny is applied to it. Therefore, as already established by previous work [7], we argue that the biases have to be comparably small and the data set is thus reliable enough to allow us to derive insights into the peering ecosystem for two reasons. Firstly, it has a very good standing in the network operators community, which naturally has very big interest in having reliable peering information available. Some of the biggest, and arguably most important organisations (e.g., Google, Netflix or Cloudflare), rely on PeeringDB. The first two refer to PeeringDB as authoritative and sole information source regarding peering capabilities. Cloudflare even automatically provisions their configuration from PeeringDB, expressing a high level of trust in PeeringDB's data. Furthermore, PeeringDB is sponsored by a multitude of large organisations (e.g., Facebook, Microsoft, Akamai), stressing the importance and usefulness of it for their network operations. Secondly, recent studies have found that PeeringDB data is consistent with BGP derived information [7] as well as with other publicly available data sources on IXPs [4]. In this paper, we are thus going to treat data from PeeringDB as a ground-truth for our analysis.

The data snapshot used in this paper was retrieved on Jan 10 , 2018.

${ }^{1}$ https://www.peeringdb.com 


\begin{tabular}{lr}
\hline Entity & Count \\
\hline IXPs & 643 \\
Organisations (total) & 11,918 \\
Organisations (at IXPs) & 6,910 \\
AS numbers (total) & 11,596 \\
AS numbers (at IXPs) & 7,171 \\
\hline
\end{tabular}

Table 1: Number of entities listed in PeeringDB.

\section{HYPERGIANTS OF THE INTERNET}

In this section we dig into the PeeringDB data to identify characteristics that differentiate today's hypergiants from other organisations.

The challenge we face is that there is neither a definition of a hypergiant nor an authoritative list of hypergiants that could be used as ground truth. Despite this limitation of the current state-ofthe-art, we will nevertheless attempt to reach a set of sufficiently convincing characteristics by relying on the fact that hypergiants are the largest among today's Internet organisations. This should make them significantly different from the majority of networks, at least across some metrics. If they were identical on all metrics, then it would either mean the data we rely on is not appropriate, or that they surprisingly aren't actually the largest among today's Internet organisations, which would then highly question the very term hypergiant originally coined in [5].

To identify such metrics, we first look at the port capacity, geographic footprint and traffic profiles of all organisations participating in the public peering landscape. We then combine these three dimensions into a single, more coherent picture and employ a clustering algorithm to separate possible hypergiants and other organisations. The cluster made of these organisations matching what is expected from hypergiants will therefore be considered as the current set of hypergiants, as visible through the PeeringDB data snapshot we used.

\subsection{The Peering Landscape}

In this subsection we use 3 dimensions to obtain a first characterisation of Internet organisations: port capacity, geographic footprint and traffic profile. Port capacity is a proxy metric for the actual amount of traffic, being a likely higher bound on the actual amount of traffic exchanged. Geographic footprint reflects the geographic deployment of organisations. Traffic profile finally is the self-declared directionality of the traffic by organisations, which differs strongly between eyeballs, transit networks, and content-heavy players of the Internet ecosystem.

Port capacity. Based on PeeringDB data, we extract for each organisation the IXPs it is present at, along with the corresponding router port sizes. We then sum up those port sizes to obtain the aggregated provisioned port capacity. The total aggregate port capacity across the data amounts to $290 \mathrm{Tbps}$, with an average port capacity of 40.45 Gbps per organisation.

One would naturally expect that hypergiants should be amongst the organisations with the highest provisioned port capacity. Figure 1 shows the port capacity provisioned by each organisation

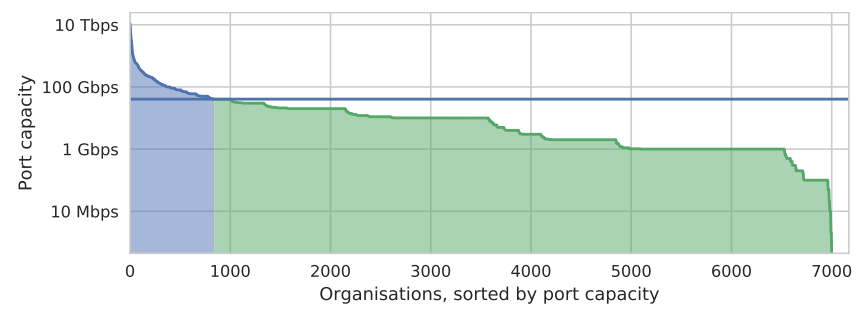

Figure 1: Total provisioned IXP port capacity for each organisation. The horizontal line depicts the average port capacity (40.45 Gbps). Organisations having more than the average capacity provisioned are depicted in blue, organisations having less than average in green. Note the log-scale of the y-axis.

present in PeeringDB. The distribution of provisioned port capacity is strongly non-uniform, with an average of $40.45 \mathrm{Gbps}$ but a standard deviation of 311.6 Gbps. Figure 1 exposes that a few organisations are responsible for a significant, way above-average port capacity (blue bars), while the overwhelming majority of them declares below-average capacity (green bars). The top five largest organisations represent $16.3 \%$ of the total port capacity, the top 80 covering half. In contrast with these massive organisations, the majority provisions significantly less port capacity. This result is in line with the traffic figures from [5].

Geographic footprint. We now turn to the geographic footprint, by looking at the number of continents ${ }^{2}$ where an organisation is present at IXPs. We expect that hypergiants will aim to have wide, if not global, geographic presence, publicly exchanging data in IXPs across multiple continents. Figure 2 shows in its columns the distribution of continent presence in the PeeringDB data set. The largest share of organisations $(6,623)$ are present on only one continent. On the other hand, there are only 89 with presence across four or more continents. Among those 89 organisations with most port capacity, the top ones, Apple (4 continents), Twitch (5 continents), Amazon (6 continents) and Google (7 continents), are strong hypergiant candidates.

Traffic profile. Organisations do not only differ in their geographic footprint and total port capacity, but also in their purpose and thus traffic profile. Some, such as content providers, are expected to have a predominantly outbound traffic profile, whereas ISPs connecting eyeballs to the Internet are expected to have an inbound traffic profile. PeeringDB defines five different profiles ranging from (Heavy) Inbound to (Heavy) Outbound, with Balanced in the middle. Organisations not wishing to expose their traffic profile have the option 'Not Disclosed' as well. ${ }^{3}$ Figure 2 shows in its rows the traffic profiles of the organisations in the data set. Besides a small fraction who hide their traffic profile, we see that the majority are inbound oriented or balanced, likely referring to eyeballs and transit networks respectively. There are more than twice as many

\footnotetext{
${ }^{2}$ PeeringDB recognises the following continents: Africa, Asia Pacific, Australia, Europe, Middle East, North America and South America. We adopt this non-textbook definition of a continent to maintain comparability to other works using PeeringDB data. ${ }^{3} \mathrm{~A}$ few organisations chose to leave the corresponding database field empty. We treat these the same as 'Not Disclosed'.
} 


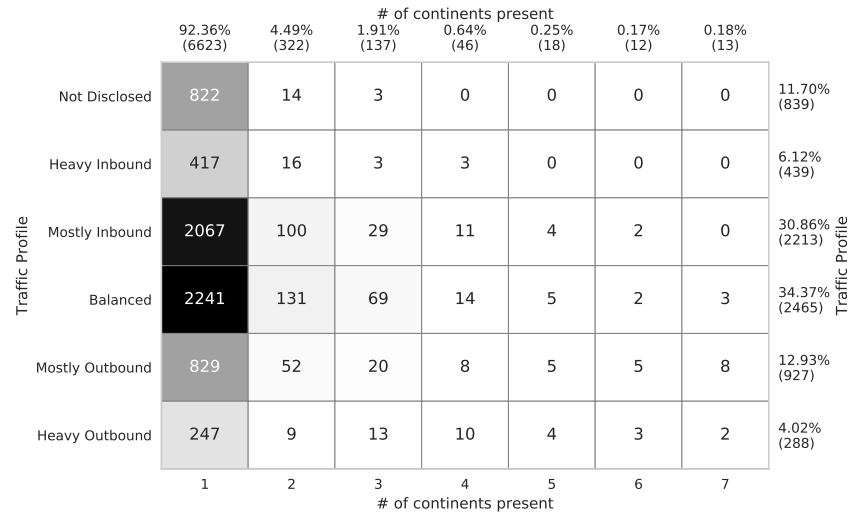

Figure 2: Traffic profile and continent coverage for each organisation. Continent presence means an organisation is present at at least one IXP of this continent. Secondary axes show the distributions of organisations across traffic profiles and continent presence.

organisations with an inbound traffic profile than with an outbound profile. Among the 288 organisations with a Heavy Outbound profile, the majority (247) have presence in a single continent, making them very unlikely to be a hypergiant. Only 41 organisations declare a Heavy Outbound profile and are present at multiple continents. 139 organisations declare an Outbound (Heavy or not) profile and are present at multiple continents. These numbers suggest that finding hypergiants among sufficiently large organisations that have extensive footprint should be possible.

\subsection{The Whole Picture}

After having discussed the three dimensions in isolation, we now put them together to obtain a more comprehensive picture of the organisations participating in the peering ecosystem, as seen from PeeringDB. Figure 3 shows a tree-map combining the three dimensions: continent presence, traffic profile, and aggregate port capacity. In this tree-map, the area of each rectangle is proportional to the aggregated port capacity it represents. Organisations are first grouped by number of continents (one to seven) at which they maintain IXP presence, enclosed by a white border. The on-print shows the number of continents of each group, and the aggregate port capacity of all its members. Each group is then subdivided by the traffic profiles of the group's organisations.

First, we observe that organisations present at a single continent account for $45 \%$ of the overall port capacity. The remaining capacity is spread almost evenly across the other groups in terms of continent presence, with between 8-13\% for each group, except for the group of five continents that has only $3 \%$. While $92 \%$ of all organisations are present at a single continent, they are only responsible for $45 \%$ of the total provisioned port capacity. In contrast, the $1 \%$ of them with presence on four continents or more are responsible for $38 \%$ of provisioned port capacity. This implies that the many organisations with a local geographic scope tend to have little port capacity (hence little expected traffic) at IXPs. In contrast, there are a few with large geographic scope, combined with large port capacity (hence large expected traffic) at IXPs.

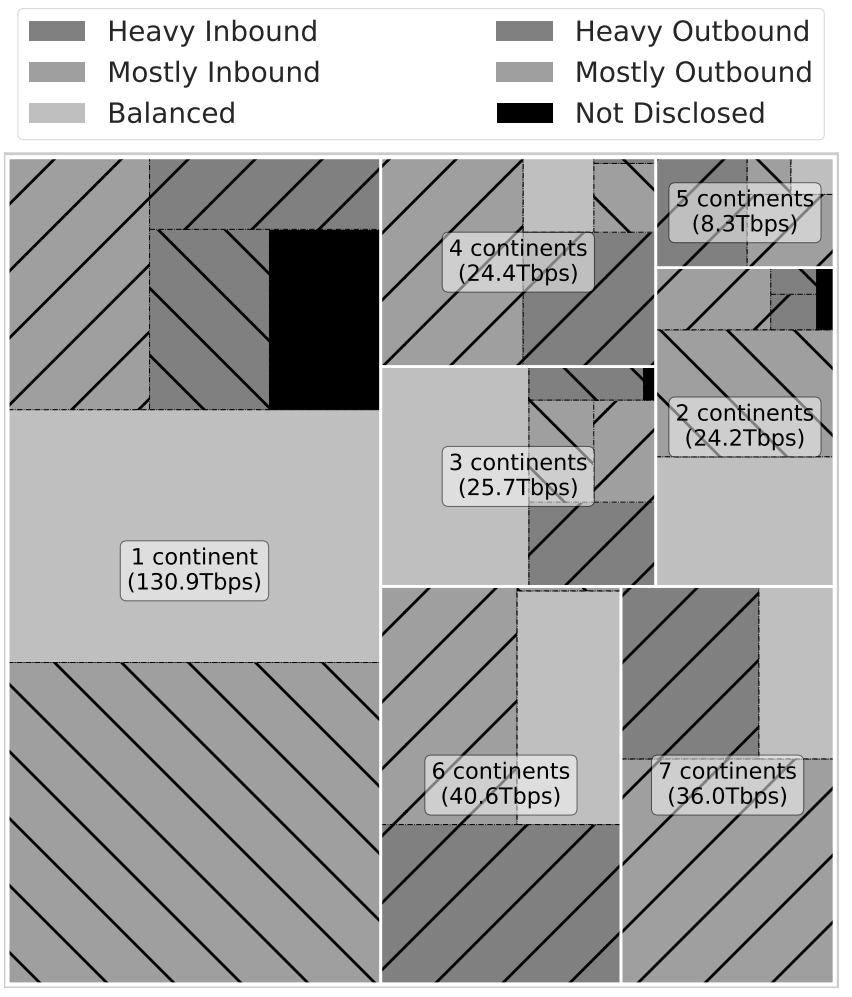

Figure 3: Distribution of aggregated port sizes over traffic profiles and continent presence. An organisation is present on a continent if it is present at an IXP at this continent. The area of each rectangle is proportional to the aggregated port size it represents. Organisations are grouped by number of continents and then by traffic profile. The on-print depicts the number of continents organisations are present at and aggregated port size of the organisations in each group.

Second, within each group of organisations in terms of continent presence, their composition differs in terms traffic profile. Within the single continent group, more than $75 \%$ of the port capacity belongs to balanced (30.6\%) or inbound dominant (45.9\%) organisations. Among the organisations in this group with an outbound traffic profile, we find content and hosting providers with a local audience, like BBC, Hetzner, Strato, VKontakte and Baidu.

Looking at the groups with presence in multiple continents, we see a smaller contribution from inbound traffic profiles to the total port capacity. While inbound dominant organisations still have a notable share in the groups of two, three and four continents, they play no role in the groups of five, six or seven continents. In those groups, organisations with an outbound traffic profile are dominant. Balanced organisations with presence at four or more continents are those with a data-centric business model, that do not only deliver but also consume content, such as Dropbox, Amazon (AWS), Hurricane Electric and Microsoft.

In this subsection, we have seen how a relatively small group of global organisations gather a substantial amount of port capacity. Moreover, they mostly declare an outbound or balanced traffic 


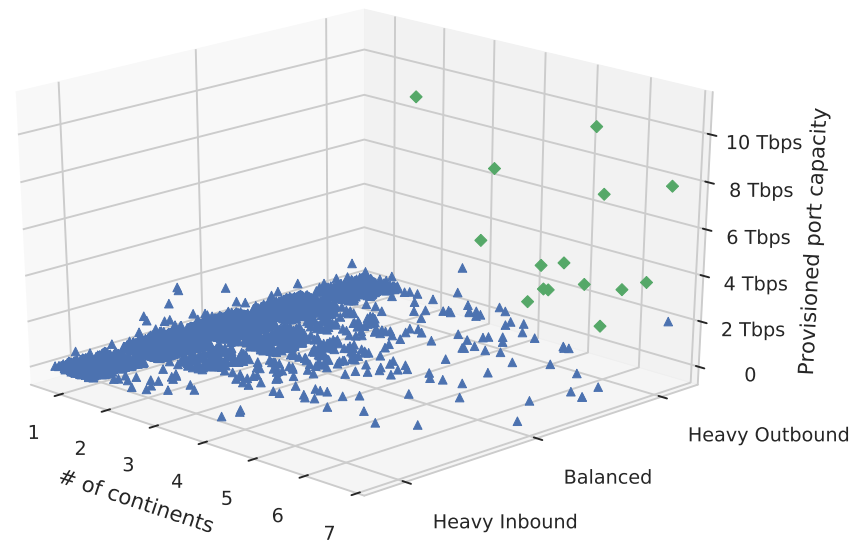

Figure 4: Results of the k-means clustering. Organisations in the smaller group are depicted by the green diamonds, those in the other group by the blue triangles. We added a small jitter on the $x$-and $y$-axis to make markers easier to discern.

profile. This is expected, as large content providers strive to deliver their content to a global audience of end customers. Based on what we observe in this section, content providers rely on a wide IXP presence to serve traffic to the eyeball organisations that operate smaller networks with a local footprint and have an inbound traffic profile. Further, this strong concentration of port capacity strongly hints at hypergiants, which are quite likely to be in this small group of global organisations.

\subsection{Hypergiants of the Internet}

Depending on their business model, hypergiants should exhibit different characteristics. Intuitively, content hypergiants are expected to be heavy on (outbound) traffic, with a large geographic reach to cater for a world-wide customer base. Cloud hypergiants will have similar characteristics, however their traffic profile might be more balanced. In general, we expect hypergiants to fall within the group of organisations with an outbound or balanced traffic profile and presence on many continents. In the following, we will try to identify a small subset of organisations fulfilling these characteristics, while being significantly different from the remaining ones.

We use the k-means algorithm [6] to split the organisations from the data set in two clusters, expecting that hypergiants are different enough to form a cluster on their own. We first use the k-means algorithm as provided by the Python module scikit-learn [10] with default values for all parameters except for the number of clusters, which we set to two. Data is preprocessed and normalised using scikit-learn's RobustScaler. The clustering yields one cluster with 15 organisations and a second cluster that contains the remaining 7,156. Figure 4 visualises the resulting clustering, with the blue triangular markers depicting the larger cluster, and the green diamond shaped markers depicting the smaller cluster. We added a small jitter on the $\mathrm{x}$ - and $\mathrm{y}$-axis to make markers easier to discern.

To ascertain that this split in the data set is not an artifact of the clustering method we employed, we apply further clustering and outlier detection algorithms to the data set. In contrast to k-means,

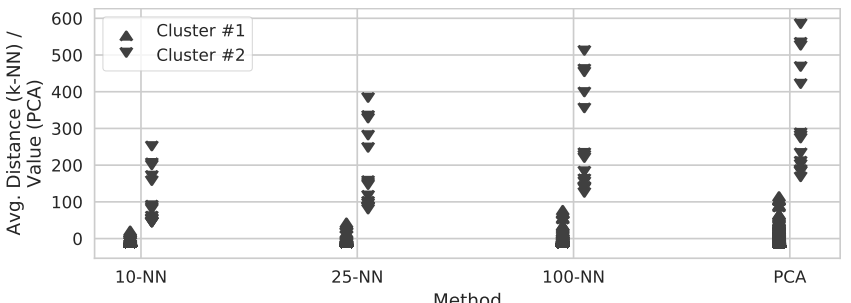

Figure 5: Data point scores assigned by k-NN and PCA. We plot data point with small horizontal offsets representing the k-means clustering we derived previously. For each method the scores assigned to cluster \#2 are always higher than the scores for datapoint in cluster \#1.

these methods do not directly cluster the data, merely providing a score for each data point. A threshold is required to translate this scoring into a clustering, but choosing the threshold value is difficult, since we do not know a priori how many outliers should be expected and there is no ground truth to be compared against.

We show results for principal component analysis (PCA) and the k-nearest neighbour algorithm (k-NN). For PCA, we chose to reduce to one principal component only and use the resulting values directly as scores, since this dimension alone captures more than $99.5 \%$ of the variance. For k-NN, we use the average distance to the 10, 25 and 100 nearest neighbours. Data was preprocessed in the same way as for the clustering. The resulting scores are shown in Figure 5. We marked data points by the original clustering obtained through k-means, and offset the two groups in the figure to ease distinguish the clusters. We observe that the resulting scores are consistent with the original k-means clustering: the top 15 scores in every ranking belong to the data points from the small cluster. This makes it possible to choose a threshold such that the resulting clustering is identical to the one obtained through $\mathrm{k}$-means.

We now take a closer look at the resulting clustering. The 15 organisations in the smaller cluster represent only $0.2 \%$ of all organisations, yet they account for more than $30 \%$ of provisioned port capacity. This smaller group does not only accumulate a disproportionately large share of port capacity, but all its members are also present on at least four continents and either have a heavy outbound or balanced traffic profile. There is a strong contrast to the other cluster, whose members are on average present on one continent only, and most of its members have an inbound $(2,652)$ or balanced $(2,463)$ traffic profile. This contrast strongly suggests that the smaller cluster captures organisations fulfilling the expectation we have for hypergiants.

Table 2 lists the 15 organisations that were singled out by the clustering algorithm. These fifteen networks indeed typically are considered to be hypergiants; Google, Akamai, Microsoft and Limelight are also explicitly mentioned as hypergiants in [5]. Since these 15 organisations are naturally separable from the remaining data set, we conclude that, given the dataset we used, these are the hypergiants in the Internet, at the time the dataset was taken. 


\begin{tabular}{|c|c|c|c|c|c|}
\hline & Organisation name & ASN & Continents & Port. Cap. & Traffic Profile \\
\hline 1 & Apple Inc & 714 & 4 & $10.960 \mathrm{Tbps}$ & Mostly Outbound \\
\hline 2 & Amazon.com & 16509 & 6 & $9.991 \mathrm{Tbps}$ & Balanced \\
\hline 3 & Facebook & 32934 & 6 & 9.840 Tbps & Heavy Outbound \\
\hline 4 & Google Inc. & 15169 & 7 & 8.741 Tbps & Mostly Outbound \\
\hline 5 & Akamai Technologies & 20940 & 7 & 7.854 Tbps & Heavy Outbound \\
\hline 6 & Yahoo! & 10310 & 6 & $5.310 \mathrm{Tbps}$ & Mostly Outbound \\
\hline 7 & Netflix & 2906 & 7 & $5.170 \mathrm{Tbps}$ & Mostly Outbound \\
\hline 8 & Hurricane Electric & 6939 & 7 & 5.037 Tbps & Balanced \\
\hline 9 & $\mathrm{OVH}$ & 16276 & 4 & 4.270 Tbps & Heavy Outbound \\
\hline 10 & Limelight Networks Global & 22822 & 6 & 3.840 Tbps & Mostly Outbound \\
\hline 11 & Microsoft & 8075 & 6 & $3.680 \mathrm{Tbps}$ & Mostly Outbound \\
\hline 12 & Twitter, Inc. & 13414 & 6 & 3.401 Tbps & Heavy Outbound \\
\hline 13 & Twitch & 46489 & 5 & 3.340 Tbps & Heavy Outbound \\
\hline 14 & Cloudflare & 13335 & 7 & 3.320 Tbps & Mostly Outbound \\
\hline 15 & Verizon Digital Media Services & 15133 & 6 & $3.030 \mathrm{Tbps}$ & Heavy Outbound \\
\hline
\end{tabular}

Table 2: The fifteen hypergiants sorted by port capacity.

\section{THE REACH OF HYPERGIANTS}

So far, our focus has been on the specific information present in PeeringDB, in a way that would help us identify hypergiants. We found out that the geographic presence was a strong aspect differentiating aspect. Combined with the traffic profile and port capacity, this led to a ranking of organisations on PeeringDB that exposes hypergiants.

Now, we slightly shift the focus onto IXPs: we ask how hypergiants rely on IXPs to build their interconnection footprint. More specifically, we would like to answer: what it is that hypergiants are looking for with their IXP presence?

Quite naturally, a hypergiant should have a strong interest to reach eyeball IP address space, as they have built their business model around providing services to end users. While this might be less critical to cloud hypergiants that are more focused on hosting networked applications and services, this is definitely very important to content hypergiants like Netflix, who generate their revenue through end-users paying for their services.

We define the potential reach of an organisation as the number of potentially reachable IP addresses through its IXP presence, by peering with the other organisations also present at the same IXPs. To compute this metric, we combine the IXP membership information from PeeringDB with Routeviews routing information and customer cones from CAIDA [8]. For every organisation, we extract all the IXPs it is present at, and then for each IXP extract all the ASes present. We then use the routing information and customer cones to map ASes to customers and IPv4 prefixes, and then calculate the number of unique IPs covered by those prefixes.

The boxplots in Figure 6 show the distribution of potential reach among the organisations. Whenever IPs are reachable through members with different peering policies, we assume they are reached through the peer with the most open peering policy, i.e., open > selective $>$ restrictive. In all boxplots, the whiskers indicate the full range of the data. The left figure shows the number of directly reachable IPs of organisations, by peering at the IXPs they are present with all the other members. We only consider IP space of
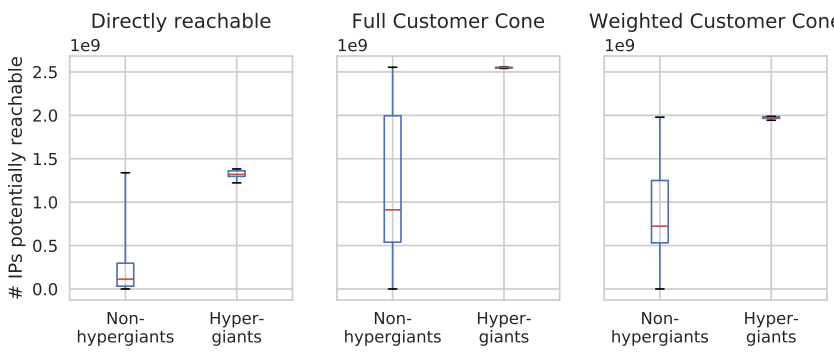

Figure 6: Potentially reachable IP space through peerings at IXPs. Whiskers show full range of the data.

the IXP members, not the customer cones. The center figure shows the potential reach, by peering with all other IXP members, assuming these members give full access to their customer cone. The right figure show the potential reach, by peering with all other IXP members, also considering peering policies of the other members. We assume that members with an open policy give access to the full customer cone; members with a selective policy to $66.6 \%$ of it; and members with a restrictive policy to only $33.3 \%$ of it. When no peering policy is stated, we assume access to $50 \%$ of the customer cone.

When we focus on reachable IP addresses for the 15 hypergiants previously identified by k-means clustering, we observe that they can indeed reach a significant amount of the address space. In all three cases we consider, all of them are among those organisations with the highest reach. While we observe that the majority of nonhypergiants have a smaller reach than the identified hypergiants, some organisations that are not clustered as hypergiant also have a similar reach. From these boxplots, we can conclude that hypergiants are in the set of organisations with the biggest reach, while at the same time there also are other organisations with a comparable reach. Additional aspects, such as port capacity (or traffic if available), and footprint are necessary to differentiate hypergiants from other organisations. 


\section{DISCUSSION}

Hypergiants. In this paper we focused on coming up with a set of characteristics that allows to identify the hypergiants coined by Labovitz et al. [5]. Because we relied on global reachability as seen through PeeringDB as a way to find these hypergiants, we limited our study to the largest of them. However, there is a variety of organisations that operate on a less global scale than these specific hypergiants, which still exchange a significant amount of traffic, without relying on a global footprint due to the nature of their business, e.g., BBC. Also, some organisations that are not considered as such yet will become hypergiants in the future. Our results only apply to the time at which the dataset we use was collected, the Internet is a fast-changing ecosystem. Further work into the diversity of hypergiant-like organisations and their evolution is needed if we are to truly understand the Internet ecosystem and its diversity.

Public vs. private. Despite the unique and rather trustworthy information provided by PeeringDB, it misses an important part of the Internet network interconnection ecosystem, namely private peerings. Some large hypergiants, such as Facebook, rely heavily on private interconnection to deliver their traffic $[11,12]$. Fortunately, despite not showing the private part of the network interconnection ecosystem, PeeringDB appears to provide sufficient information to still see the largest hypergiants. However, PeeringDB provides a view that (largely) underestimates the network interconnection ecosystem of the Internet. This bias is similar to the one of the AS-level topology, for which publicly available BGP routing data misses a large fraction of the AS-level connectivity [9], especially due to the rich worldwide IXP ecosystem $[1,2]$.

\section{RELATED WORK}

In their seminal work, Labovitz et al. [5] were the first to coin the term hypergiant. They observed a shift over time of traffic being diverted away from large Tier-1 and Tier-2 backbone networks and instead being directly exchanged between networks without any intermediary. This observation forced the research community to significantly revisiting their mental model of the Internet. Our work is motivated by their use of the word hypergiant, which is currently lacking a precise definition. In contrast to their work, we do not use traffic measurements but information within PeeringDB augmented by routing information to characterise hypergiants.

Previous works have used PeeringDB as an information source, extracting insights about the peering ecosystem and assessing its usability to better understand the Internet ecosystem. Lodhi et al. [7] made a first step in assessing the reliability and thus usability of PeeringDB for Internet research. They assessed the plausibility of PeeringDB data by comparing the information in PeeringDB against Local Internet Registries (LIRs) and BGP data. They found that while the data exhibits some biases, overall it appears to be reliable. They also made a first attempt at characterising the participating organisations. In contrast to our work, their focus is more on an overall assessment of PeeringDB than on analysing organisations specifically. Klöti et al. [4] compared the data in PeeringDB against data from other publicly available IXP data sets. They linked together the data sets available from PeeringDB, Euro-IX and $\mathrm{PCH}$ to assess their degree of complementarity and completeness. While they found biases in every data set, caused by its sourcing and intended usage, they nevertheless concluded that the data sets present similar views of the Internet.

\section{SUMMARY}

In this paper we combined PeeringDB and Routeviews BGP data to obtain a better understanding of today's hypergiants. Starting with a characterisation of the organisations taking part in public traffic exchange, we identified features differentiating hypergiants from the other organisations. Based on these features, we identified fifteen hypergiants. We then explored whether the approach those hypergiants take to make use of IXPs to reach their global customer base is unique. While it is different to many of the other organisations, on its own it not is sufficient to differntiate hypergiants from all other organisations. All these steps identified and discussed important characteristics of hypergiants, a set of organisations which has a significant impact on the Internet, due to the massive amount of traffic they are responsible for.

\section{REFERENCES}

[1] Bernhard Ager, Nikolaos Chatzis, Anja Feldmann, Nadi Sarrar, Steve Uhlig, and Walter Willinger. 2012. Anatomy of a large european IXP. In Proc. of SIGCOMM. ACM.

[2] Nikolaos Chatzis, Georgios Smaragdakis, Anja Feldmann, and Walter Willinger. 2015. Quo vadis Open-IX? Computer Communication Review 45, 1 (2015).

[3] Sushant Jain, Alok Kumar, Subhasree Mandal, Joon Ong, Leon Poutievski, Arjun Singh, Subbaiah Venkata, Jim Wanderer, Junlan Zhou, Min Zhu, Jon Zolla, Urs Hölzle, Stephen Stuart, and Amin Vahdat. 2013. B4: experience with a globallydeployed software defined wan. In Proc. of SIGCOMM. ACM.

[4] Rowan Klöti, Bernhard Ager, Vasileios Kotronis, George Nomikos, and Xenofontas A. Dimitropoulos. 2016. A Comparative Look into Public IXP Datasets. Computer Communication Review 46, 1 (2016).

[5] Craig Labovitz, Scott Iekel-Johnson, Danny McPherson, Jon Oberheide, and Farnam Jahanian. 2010. Internet inter-domain traffic. In Proc. of SIGCOMM. ACM.

[6] Stuart Lloyd. 1982. Least squares quantization in PCM. IEEE transactions on information theory 28, 2 (1982).

[7] Aemen Lodhi, Natalie Larson, Amogh Dhamdhere, Constantine Dovrolis, and kc claffy. 2014. Using peeringDB to understand the peering ecosystem. Computer Communication Review 44, 2 (2014).

[8] Matthew J. Luckie, Bradley Huffaker, Amogh Dhamdhere, Vasileios Giotsas, and kc claffy. 2013. AS relationships, customer cones, and validation. In Proc. of IMC. ACM.

[9] Ricardo V. Oliveira, Dan Pei, Walter Willinger, Beichuan Zhang, and Lixia Zhang. 2010. The (in)completeness of the observed internet AS-level structure. IEEE/ACM Transactions on Networking 18, 1 (2010).

[10] F. Pedregosa, G. Varoquaux, A. Gramfort, V. Michel, B. Thirion, O. Grisel, M. Blondel, P. Prettenhofer, R. Weiss, V. Dubourg, J. Vanderplas, A. Passos, D. Cournapeau, M. Brucher, M. Perrot, and E. Duchesnay. 2011. Scikit-learn: Machine Learning in Python. Journal of Machine Learning Research 12 (2011), 2825-2830.

[11] Arjun Roy, Hongyi Zeng, Jasmeet Bagga, George Porter, and Alex C. Snoeren. 2015. Inside the Social Network's (Datacenter) Network. In Proc. of SIGCOMM. ACM.

[12] Brandon Schlinker, Hyojeong Kim, Timothy Cui, Ethan Katz-Bassett, Harsha V. Madhyastha, Ítalo Cunha, James Quinn, Saif Hasan, Petr Lapukhov, and Hongyi Zeng. 2017. Engineering Egress with Edge Fabric: Steering Oceans of Content to the World. In Proc. of SIGCOMM. ACM. 